\title{
La inclusión del médico en la formación del intérprete en el ámbito sanitario
}

\author{
The Inclusion of the Doctor in the Training of the Interpreter in Healthcare \\ A inclusão do médico na formação do intérprete no âmbito sanitário
}

Raquel Sanz-Moreno* (https://orcid.org/0000-0002-0861-6665)

Recibido: $12-09-17$

Departamento de Teoría de los Lenguajes y la Comunicación, Facultad de Traducción,

Revisado: $27-10-17$

Filología y Comunicación, Universidad de Valencia, Valencia, España.

Aceptado: $15-11-17$

Publicado: 18-12-17

RESUMEN. La experiencia docente que describimos en este artículo propone la adquisición y desarrollo de destrezas interpretativas a través de cuatro sesiones teórico-prácticas sobre interpretación en los servicios públicos (ISP). Su particularidad reside en integrar a un profesional del ámbito médico-sanitario en el proceso de aprendizaje, que se perfecciona mediante role plays en el aula. Su colaboración desinteresada, activa y participativa estuvo presente desde la elección de los casos que se abordaron en clase derivados de sus experiencias con pacientes extranjeros, hasta la asunción del papel que habitualmente desempeñan en su trabajo en los ejercicios de simulación con los alumnos. La interacción con los médicos no solo contribuyó a dar mayor realismo y autenticidad al role play, sino que favoreció el desarrollo de destrezas temáticas, interpersonales y estratégicas por parte del alumnado.

ABSTRACT. The didactic experience we describe in this article aims at the acquisition and development of interpreting skills through four theoretical and practical sessions on Public Service Interpreting (PSI). This course is particular given that a healthcare professional takes part in the learning process, which is

Palabras clave:

Interpretación en los servicios

públicos,

interpretación

médica,

role play,

socialización con el experto
Keywords: public service interpreting, healthcare 
perfected with role-play exercises as a useful tool. The generous, active, and participative collaboration of these professionals had an influence both in the election of the cases analyzed in class - which were based on their own experiences with foreign patients-and in the assumption of their own professional roles

interpreting, role play, socialization with the expert in the dramatization exercises with students. The interaction with the doctors contributed to providing more realism and authenticity to the role-play activity and also facilitated the development of thematic, interpersonal, and strategic skills in students.

RESUMO. A experiência docente que descrevemos neste artigo busca a aquisição e desenvolvimento das destrezas interpretativas através de quatro sessões teóricopráticas sobre interpretação nos serviços públicos (ISP). Sua particularidade reside na integração de um profissional do âmbito médico-sanitário no processo de aprendizagem, que é aperfeiçoado por meio de dramatizações na sala de aula. Sua colaboração desinteressada, ativa e participativa permeabilizou desde a seleção dos casos abordados na aula, decorrentes das experiências do médico com pacientes estrangeiros, até a (assunção - presunção) do papel que

Palavras-chave: Interpretação nos serviços públicos, interpretação médica, dramatizações socialização com o especialista normalmente desempenham em seu trabalho nos exercícios de simulação com os alunos. A interação com os médicos não apenas contribuiu a dar maior realismo e autenticidade à dramatização, mas também favoreceu o desenvolvimento de destrezas temáticas, interpessoais e estratégicas por parte dos alunos

La interpretación en los servicios públicos (ISP) admite una diversidad de denominaciones (ValeroGarcés, 2006, p. 37), que son un reflejo de las dificultades que existen en cuanto a la delimitación de su campo de acción y de su definición. En este sentido, entendemos la ISP como una modalidad de interpretación que tiene lugar entre personas que no hablan la lengua del país en el que se encuentran por diversas circunstancias (trabajo, turismo, estudios, etc.), y el personal que colabora en los servicios públicos, como la sanidad, la educación, los servicios sociales y la administración de justicia (Martin, 2014, párr.1). Para que estos servicios se proporcionen de forma óptima es conveniente contar con la intervención del intérprete, cuya principal función es la de facilitar la comunicación, no solamente lingüística, sino también cultural, entre los intervinientes en la situación comunicativa.

La interpretación en estos contextos se perfila como un elemento esencial para garantizar la calidad del servicio que se presta, por lo que estimamos indispensable que el futuro intérprete no solo se familiarice con el mismo, sino que además conozca de primera mano cómo funcionan esos servicios públicos en los que se integrará cuando acceda al mercado laboral. Sin embargo, tal y como afirma Angelelli (2008), no podemos olvidar que el intérprete “is capable of processing and conveying 
information in two languages, often under conditions of critical and extreme pressure" (p. 148). Por esta razón, una formación sólida y en contacto con la realidad de la práctica de la ISP es fundamental para que el alumno acceda al mercado laboral teniendo referencias lo más reales posibles acerca de lo que puede encontrarse en él.

Nuestra intención es facilitar la adquisición de destrezas interpretativas por parte del estudiantado, entendidas como "the combination of aptitudes, knowledge, behaviour and know-how necessary to carry out a given task under given conditions" (Medlnt, 2008, p. 4). Para ello, partimos del proyecto Medlnt (2008) que desarrolla un modelo curricular basado en una serie de competencias indispensables para todo intérprete especializado en el ámbito médico-sanitario, que se desglosan en seis grandes bloques: competencias interpretativas (tanto profesionales como interpersonales), interculturales, lingüísticas, tecnológicas, temáticas y de búsqueda de información. Dado el breve tiempo del que disponíamos para desarrollar este proyecto, nuestro objetivo era motivar al alumno y dar a conocer la realidad del trabajo diario de un intérprete y de los profesionales de la Medicina en este contexto, de forma que consiguiera tener una mayor conciencia del carácter fundamental de su papel en la interacción con el profesional en el ámbito sanitario y erigirse como "powerful co-participants who possess agency in the interaction" (Angelelli, 2008, p. 159).

A lo largo del presente artículo recordaremos brevemente las ventajas de contar con expertos en la materia en el aula de interpretación, así como la utilización de los role plays como herramientas didácticas de gran utilidad en estos ámbitos; además, señalaremos algunas experiencias docentes en la interpretación médica que nos han servido como antecedente del proyecto que presentamos. Por último, describiremos nuestro proyecto, ahondando en sus objetivos, su contexto, así como en los resultados obtenidos.

\section{La socialización con el experto en la materia}

La ISP es una de las modalidades de interpretación en la que los intérpretes deben interactuar con expertos de distintas materias y facilitar la comunicación entre estos y los destinatarios de los servicios. A diferencia de la traducción, el contacto con los profesionales en la ISP es muy directo, comparten espacio físico y participan en el intercambio conversacional con ellos. El intérprete no solo entra en contacto con los profesionales, sino que además accede a sus entornos físicos de trabajo y forma parte activa de la dinámica que se genera en dichos contextos. Así, y dado que los expertos en Derecho o en Medicina, por poner dos ejemplos, disponen de unas normas y convenciones que les son propias, y que no solo se manifiestan en sus escritos sino también, y muy especialmente, en la forma en la que se expresan oralmente, el intérprete debe familiarizarse con estas convenciones para perfeccionar su interpretación. La socialización terciaria (Monzó, 2003) se presenta como un elemento fundamental en el desarrollo profesional de las destrezas del traductor $y$, entendemos, también del intérprete. A diferencia de la socialización secundaria que convierte al traductor "en un agente social del campo de la mediación cultural", la socialización terciaria "se realiza de un modo más consciente, selectivo y manipulativo" (Monzó, 2003, p. 20), lo que implica que el traductor/intérprete se adentre de forma consciente en la comunidad de expertos en la que va a trabajar y conozca de primera mano las condiciones reales que imperan en ese contexto laboral. Los traductores especializados deben apropiarse de las convenciones y conocimientos de los que disponen los expertos en la materia para poder asegurar 
una mayor comprensión de cómo funciona la comunicación en estos contextos (Montalt, 2005, p. 23). Para ello, el trabajo cooperativo y la colaboración entre agentes intervinientes en la interacción interpretativa es fundamental para el desarrollo y consolidación de esta socialización, teniendo en cuenta que los expertos en Medicina, en este caso, no constituyen únicamente una comunidad de conocimiento receptora de la interpretación, sino también parte activa de la misma. Ahora bien, es indudable que el alumno de ISP no tiene un fácil acceso a la comunidad de expertos, por lo que el aula se perfila como el primer espacio en el que se puede facilitar el intercambio entre ambas partes en un contexto más relajado y en el que la interacción se desarrolla con unos parámetros controlados.

\section{Role plays en interpretación}

El role play consiste en una dinámica de grupo en la que dos o más personas representan una situación, actuando según el papel que se les ha asignado. Presenta numerosas ventajas en el aula, como observar la reacción de los alumnos frente a una situación determinada, calibrar las consecuencias de sus decisiones, darse cuenta de que no hay una única respuesta o una respuesta correcta, sino que existen disparidad de actitudes que se pueden adoptar, y además permite experimentar en un ambiente con riesgo limitado. Esta herramienta didáctica se viene empleando tradicionalmente en el aula de ISP. Así, Fernández Pérez $(2011,2015)$ emplea ejercicios de simulación en la didáctica de la interpretación telefónica; Baigorri et al. (2006) y Alonso y Baigorri (2008) también los utilizan como materiales didácticos en la ISP; Ozolins (2013) explica un interesante proyecto de role play en ISP de una niña china encontrada en Australia sin ningún dato que pudiera arrojar luz sobre su procedencia. Lázaro (2007) y Jiménez Ivars (2012) también emplean los role plays en contextos médicos, jurídicos o sociales.

La novedad del proyecto que presentamos radica en la inclusión del experto en la materia en el aula de interpretación. Este cambio supera las dificultades observadas en role plays realizados únicamente por alumnos. Dado que estos no poseen las convenciones ni los conocimientos que detentan los expertos, los role plays con guiones previos son "menos apropiados para la adquisición de destrezas relacionadas con la coordinación de la interacción conversacional real”, y aunque la profesora hubiera escrito los textos, la interacción perdería espontaneidad y naturalidad, ya que los alumnos suelen acabar leyendo el texto propuesto (Fernández Pérez, 2015, p. 263). Por otra parte, en las simulaciones improvisadas, los alumnos tienden a realizar intervenciones breves y "con menor densidad conceptual" (Fernández Pérez, 2015), dado su desconocimiento temático y terminológico. Cuando se cuenta con un profesional de la materia, este utiliza una jerga especializada propia, no impostada y que emplea a diario, a menudo sin ser consciente de su dificultad. La naturalidad y la espontaneidad, en este caso, están garantizadas, ya que el experto posee los conocimientos especializados que requiere la situación comunicativa y está acostumbrado a actuar en estas situaciones. Esto, obviamente, no se puede dar cuando el alumno adopta el papel de médico o de abogado en un ejercicio de simulación. Además, el profesional conoce perfectamente las distintas eventualidades que pueden surgir de las interacciones entre médico-paciente o abogado-cliente, ya que los casos que se abordaron con los alumnos en este proyecto provenían de situaciones reales experimentadas por los profesionales. Por tanto, contar con el experto en clase favorece la adquisición de destrezas comunicativas relacionadas con el desarrollo de la interacción (turnos de palabras, interrupciones, lenguaje no verbal etc.), destrezas relacionadas con el manejo de terminología especializada o una toma de notas más eficaz (Fernández Pérez, 2011, p. 165). 


\section{La didáctica de la interpretación en el ámbito médico-sanitario}

La interpretación en el ámbito médico-sanitario forma parte de algunos programas de máster en ISP (Máster Universitario en Comunicación Intercultural, Interpretación y Traducción en los Servicios Públicos, de la Universidad de Alcalá de Henares, Máster en Traducción, Interpretación y Estudios Culturales, de la Universitat Autònoma de Barcelona), en interpretación de conferencias (Máster de Interpretación de Conferencias, Universidad Europea de Madrid; Máster on-line en Interpretación de Conferencias de Medicina y Odontología, Universidad Católica de Murcial o en Traducción médica (Máster Universitario en Traducción Médico-Sanitaria, Universitat Jaume I).

En la mayoría de los casos se dedica un número limitado de créditos a familiarizarse con el contexto médico-sanitario y con las distintas modalidades de interpretación que pueden emplearse en él (interpretación consecutiva monológica, dialógica, simultánea y traducción a la vista). Las metodologías de enseñanza y aprendizaje se basan en “la participación activa de los alumnos e intercambios de experiencias relacionadas con la traducción e interpretación en el ámbito sanitario (...), role plays y grabaciones específicas para situaciones reales" (Universidad de Alcalá de Henares); conferencias de profesionales externos de los diferentes ámbitos de especialización (entre los que se incluyen el médico), con el fin de que el alumno establezca un vínculo directo con la práctica profesional (Universidad Autónoma de Barcelona); o “formación especializada a cargo de jefes de Servicio, de Sección y Médicos adjuntos de hospitales de referencia" (Universidad Europea de Madrid). Como podemos comprobar, existe una preocupación real por dar al alumno una imagen lo más fiel posible de la realidad del ejercicio de su futura profesión y predomina el aspecto práctico a la hora de impartir estas clases que, de manera general, se dan a nivel de máster.

Lázaro y Pena (2010) describen la formación de los intérpretes en el ámbito pediátrico y Vitalaru y Lázaro (2011) en interpretación sanitaria en general en el marco de FITISPOS, grupo dedicado a la investigación y formación en traducción e ISP. También el Grupo CRITT (2014) de la Universitat Jaume I de Castellón estudia la práctica de la mediación interlingüística e intercultural en el ámbito sanitario, y se centra en dos ámbitos específicos (salud mental y salud reproductiva), describiendo las intervenciones de mediadores del propio grupo en hospitales de Castellón y Valencia. Los casos que se describen en estos trabajos, así como las observaciones realizadas por los autores, nos han servido como antecedentes para preparar las sesiones que conforman este proyecto aunque, como hemos adelantado, la mayor parte de casos abordados en clase provenían de la experiencia de los profesionales médicos.

\section{PROPUESTA DIDÁCTICA}

\section{Contexto}

La asignatura Técnicas y Prácticas de Interpretación Francés-Español-Catalán (I), que se imparte en el cuarto curso del grado de Traducción y Mediación Interlingüística de la Universitat de València, persigue la adquisición de destrezas interpretativas por parte del alumnado en un breve periodo lun semestre) en el que el estudiante debe familiarizarse con la traducción a la vista ${ }^{1}$ (materia sobre la

\footnotetext{
1 Entendida como la "reformulación oral en lengua de llegada de un texto escrito en lengua de partida" (Jiménez Ivars \& Hurtado Albir, 2003, p. 47). Las autoras distinguen esencialmente dos funciones: la comunicativa, que consiste en una modalidad de interpretación, pues se trata de traducir oralmente in situ para un receptor, y la instrumental, entendiendo la traducción a la vista como un medio para la obtención de una traducción escrita, o como elemento empleado en la enseñanza de la traducción.
} 
que versa el examen prácticol y, en menor medida, con la ISP. La asignatura pretende ofrecer una primera aproximación a las distintas modalidades de interpretación y animar al alumnado a que profundice en su estudio y su práctica.

Dentro de la modalidad de ISP que se enseña en clase, se dedican cuatro sesiones a la interpretación en el ámbito jurídico o judicial, en las que se sigue la misma metodología didáctica (Sanz-Moreno, en prensa). No obstante, y dado que la interpretación social en el ámbito sanitario es la que mayor crecimiento ha experimentado en los últimos años (Ruiz Rosendo, 2007, p. 173), también se dedican sendas sesiones a abordarla desde una perspectiva eminentemente práctica.

El proyecto que presentamos pretendía reflejar el proceso real de trabajo de un intérprete en distintos servicios de un hospital. Por ello, lo segmentamos en tres fases. A los alumnos se les informaba con una semana de antelación de la realización de una ISP en un hospital, y se les comunicaba la especialidad en la que debían prestar sus servicios: la primera vez debían desarrollar su trabajo en una consulta del servicio de ginecología y obstetricia, en la que asistirían a la Dra. Ester Ortiz. En la segunda ocasión, debían prestar asistencia en la Unidad de Cuidados Intensivos (UCI) y trabajar con el Dr. Rafael Faus ${ }^{2}$. Se recordó a los alumnos que, aunque de manera general debían realizar una interpretación de enlace, era probable que los doctores les pidieran realizar una TAV de documentos técnicos tanto para familiares o pacientes (por ejemplo, una analítica de sangre o un historial clínico) como para el propio médico (documentos de identificación de pacientes).

Dada la labor de documentación previa que debían realizar, los alumnos se organizaron en siete grupos: cuatro de dos componentes y tres de tres componentes. Ninguno de los 17 alumnos había realizado anteriormente una interpretación en el ámbito médico-sanitario. Con carácter previo a la tercera y cuarta sesión de role plays con expertos en Medicina, la profesora dedicó dos clases teóricas para explicar las diferentes concepciones de imparcialidad en ISP y los modelos de intervención descritos por Cambridge (2002), Niska (2002) y Wadensjö (1995).

\section{Objetivos de aprendizaje}

Como ya hemos adelantado, diseñamos este proyecto teniendo en mente el desarrollo de tres tipos de competencias necesarias en la interpretación en el ámbito médico-sanitario: (1) competencias sobre búsqueda de información (búsqueda y valoración de la calidad de documentación utilizada), (2) competencias interpretativas y (3) competencias temáticas.

- Competencia sobre búsqueda de información (fase de preparación): capacidad de trabajo en equipo, documentación, búsqueda de fuentes y evaluación crítica de las fuentes de información consultadas. Asimismo, después de la interacción con el médico, los alumnos debían analizar la utilidad de los instrumentos de trabajo desarrollados (en particular, glosarios) y evaluarlos de forma crítica.

- Competencias interpretativas: desarrollo de estrategias de colaboración con el profesional de la medicina durante el role play. El alumno debía cumplir unas instrucciones dadas por un

\footnotetext{
2 Ambos profesionales trabajan en el Hospital Universitario Dr. Peset de Valencia. Participaron de forma voluntaria y altruista en la preparación de ambas sesiones y en la realización de los role plays con los alumnos. Ninguno de los dos habla francés.
} 
profesional médico, asumir y cumplir unos plazos, y actuar como si fuera un profesional, lo que modificó su visión sobre el papel real del intérprete y aumentó su conciencia respecto de la función social que este desempeña.

- Competencia temática: dado el escaso tiempo del que disponíamos, circunscribimos el ámbito temático atendiendo a los dos profesionales médicos que se prestaron a participar en los role plays: ginecología y obstetricia, y medicina intensiva. En la fase de role play, los alumnos debían demostrar los conocimientos que habían adquirido, ponerlos en práctica, interactuar con el profesional, así como poner de manifiesto su capacidad de síntesis y de análisis

\section{DESCRIPCIÓN DE LAS DISTINTAS FASES DEL PROYECTO}

\section{Primera fase: documentación previa a la interpretación.}

Dado que este proyecto se desarrolla desde hace varios años, y que la mayoría de alumnos suele manifestar que una de las preocupaciones a la hora de abordar cualquier especialidad de ISP es la precisión terminológica, en este curso decidimos complementar la práctica que habitualmente se realizaba con el experto en la materia con una fase de preparación previa tutelada. Esto nos permitía trabajar la TAV preparada (Jiménez Ivars \& Hurtado Albir, 2003, p. 50), en la que el alumnado debe traducir un texto que ha podido leer antes de la situación de uso. Según estas autoras, a mayor tiempo de lectura y preparación, mayor calidad de la reformulación. Añadimos, también, que la preparación de la TAV reduce considerablemente el nivel de estrés de los alumnos, al enfrentarse a un texto que ya conocen, que han podido analizar y del que han extraído la terminología problemática. Este ejercicio suponía igualmente una preparación a la TAV sintética (resumen oral de un texto escrito) y/o explicativa (reformulación oral de textos cortos, más bien instructivos), por lo que permitía que el alumno se familiarizara con estas modalidades desde un punto de vista práctico, experimentando las dificultades y ventajas que cada una presenta. Para ello, la profesora les entregó un pequeño dossier con textos en francés sobre normas de funcionamiento de una $\mathrm{UCl}$ e instrucciones preparatorias de una operación ginecológica con laparoscopia. Asimismo, la profesora dedicó una sesión teórica previa a la explicación de la TAV y sus diferentes submodalidades.

Las instrucciones que se les dio a los alumnos fueron las siguientes: debían organizarse en grupos de dos o tres personas para preparar la sesión. Para afrontar los ejercicios de simulación, podían llevar a la sesión documentación en papel e incluso gadgets tecnológicos como tabletas o móviles inteligentes. Se les recordó la importancia de la elaboración de glosarios en la ISP (Del Pozo, 2009; Gallego \& Tolosa, 2012), y se les invitó a trabajar en ellos, aunque no se les dio ninguna instrucción específica al respecto. Una vez conformados los grupos de trabajo, los alumnos debían dar cuenta por escrito de todo el proceso de preparación. Para facilitarles la tarea se les presentó un cuestionario que debían rellenar, que constaba de once preguntas (abiertas, de opción múltiple y dicotómicas), cuya finalidad era que el alumno reflexionara sobre su preparación para realizar este tipo de interpretación. Además se les entregó un modelo de plantilla para evaluar recursos electrónicos (ver Apéndice $A$, adaptado de Gonzalo García, Fraile \& Pérez, 2005), con el fin de que determinaran la fiabilidad de aquellos que habían consultado. 


\section{Segunda fase: Role play con el especialista en Medicina}

Durante las sesiones prácticas de tres horas de duración cada una, los estudiantes asumían los papeles de intérpretes o de pacientes ficticios, y los expertos adoptaban su papel como médico especialista en ginecología o en medicina intensiva respectivamente.

Las sesiones se articularon a través de casos prácticos en los que la ginecóloga y el intensivista, por una parte, y el alumno-paciente, por otra, recibían instrucciones sobre el caso que se abordaba, mientras que el intérprete no recibía ninguna instrucción específica. Se entregaban tarjetas con la descripción de la situación y el papel que debían desempeñar. La mayoría de casos provenían de experiencias profesionales que los médicos habían vivido en el hospital con pacientes extranjeros, por lo que se garantizaba el realismo y el conocimiento en primera persona de lo que sucedió. Los casos se abordaban una única vez y por un solo intérprete en cada ocasión. La duración no solía sobrepasar los 15 minutos. Además, las interacciones se grababan con cámara de vídeo, con el fin de poder analizarlas en clases posteriores y evaluar aspectos que en ese momento no podíamos por cuestiones de tiempo, como el lenguaje no verbal o los turnos de palabra. Los alumnos que no participaban de forma activa en el role play, asumían el papel de espectadores privilegiados que, con la distancia, realizaban un análisis crítico de las decisiones adoptadas por sus compañeros. Una vez finalizada la interacción, se iniciaba un turno de preguntas y un debate sobre lo que acababa de suceder.

\section{Tercera fase: Valoración del desempeño y de la experiencia didáctica}

En esta última fase, el alumno debía rellenar un segundo cuestionario (ver Apéndice B) de 19 preguntas en el que no solo debía valorar de 0 a 5 las destrezas y habilidades necesarias para realizar la ISP en el contexto médico-sanitario descritas por García-Beyaert (2013) (lingüística, conocimiento cultural, curiosidad, memoria, precisión terminológica, empatía, imparcialidad, secreto profesional y confidencialidad), sino que además debían indicar aquello que más les preocupaba a la hora de enfrentarse a este tipo de interpretación. En el mismo sentido, se les hacía reflexionar sobre su preparación para la sesión, realizando una valoración crítica de los instrumentos de trabajo de los que se habían servido: la plantilla de evaluación de los recursos electrónicos y el glosario. Por último, los alumnos debían valorar la experiencia de contar con un profesional médico en el aula e indicar si esta había modificado su idea de la interpretación en el ámbito médico.

\section{RESULTADOS: DESARROLLO Y VALORACIÓN DE LA EXPERIENCIA DIDÁCTICA}

En este apartado presentamos las valoraciones de la experiencia por parte del alumnado recogidas en los cuestionarios que se les hizo llegar al terminar el proyecto.

\section{PREPARACIÓN PREVIA. DOCUMENTACIÓN PARA INTERPRETAR EN SERVICIOS PÚBLICOS Aspectos lingüísticos.}

En cuanto a la preparación previa, todos los grupos manifestaron que habían leído sobre cada especialidad, mientras que seis grupos consultaron bases de datos y cinco buscaron terminología en internet. Solo un grupo recurrió a la consulta con un experto en la materia len este caso, una 
enfermeral. Cuatro de los grupos buscaron textos paralelos y comparables en francés y en español, pero como se dedujo de la memoria que presentaron, finalmente no los utilizaron.

En cuanto a las fuentes de información consultadas, los alumnos optaron por fuentes en internet. Entre estas fuentes, encontramos diccionarios en línea (monolingües y bilingües), enciclopedias en línea y glosarios elaborados por otros autores. Entre los diccionarios más utilizados, encontramos el Diccionario de la Real Academia de la lengua Española (DRAE online), el diccionario Larousse, Le Petit dictionnaire de terminologie médicale ${ }^{3}$ y Le Dictionnaire français du médicament ${ }^{4}$, todos ellos diccionarios monolingües que sirvieron al alumnado para entender los conceptos que se podían manejar en esos contextos. Una vez localizadas las fuentes de información, los alumnos debían determinar su fiabilidad utilizando la plantilla de evaluación adaptada de Gonzalo García et al. (2005) y que la mayoría de alumnos encontraron muy útil, a pesar de que no la conocían antes de realizar este ejercicio. En su opinión se trató de una herramienta que permitía una evaluación objetiva, sistemática y de calidad de los recursos electrónicos encontrados. Dos grupos $(G 2, G 4)$ manifestaron que la plantilla de evaluación les ayudó a descartar algunas propuestas de traducciones que encontraron en la red, por resultar la fuente de información poco fiable; y otro grupo (G5) expresó su satisfacción por poder justificar sus opciones de traducción frente al paciente o al médico gracias a la plantilla.

La labor de búsqueda terminológica se materializó en la elaboración de un glosario. Dado que una de las mayores dificultades que presenta la ISP en el ámbito médico-sanitario es el uso de terminología especializada y vocabulario específico (Valero-Garcés, 2005), el glosario se presenta como una herramienta muy útil no solo para el intérprete profesional sino especialmente para el alumno en formación de cara a la propia interacción con el profesional y como parte fundamental de la preparación de esta.

Todos los equipos elaboraron glosarios que fueron mayoritariamente bilingües, y en los que se incluyeron, en el $90 \%$ de los casos, la traducción del término, y en un $70 \%$ una definición. Ningún alumno incluyó una imagen o un comentario, lo que da cuenta de la preocupación del alumnado por la precisión terminológica y la necesidad de encontrar un equivalente en la lengua de llegada de los términos, prevaleciendo aquella frente a otras formas alternativas de comprensión. Los alumnos confesaron que querían que el glosario contuviera únicamente la información que les resultara útil en el momento de la interacción, por lo que todo lo demás podría resultar innecesario, o incluso molesto, a la hora de encontrar rápidamente la equivalencia del término que buscaban. El que se incluyera también una definición del término indica el afán por entender el significado de las palabras y una toma de conciencia de la necesidad de comprensión antes de abordar la interpretación. No obstante, los alumnos tampoco indicaron la fuente de la que provenía la traducción o el propio término utilizado en un breve contexto. Los glosarios contenían una media de entre 50 y 150 términos. Cinco grupos los ordenaron alfabéticamente mientras que dos lo hicieron en bloques temáticos (tratamientos, partes del cuerpo, enfermedades etc.). Los alumnos se sirvieron del software de Microsoft Excel para elaborarlo.

\footnotetext{
${ }^{3}$ https://goo.gl/Hye9PI

${ }^{4} \mathrm{https}: / / g 00 . \mathrm{gl} / \mathrm{snh} 6 \mathrm{p}$
} 
Seis de los siete grupos tenían claro que acudirían a la sesión de ISP con el glosario que habían preparado, mientras que el grupo restante no iba a llevarlo porque estaba seguro de que no lo iba a usar durante la interacción con el médico. A pesar de ello, cuatro grupos consideraban que el glosario les sería de mucha utilidad, frente a dos que albergaban dudas sobre esta y uno que no estaba seguro, por lo que prefería llevarlo a la sesión "por si acaso". Las razones que adujeron mayoritariamente para no utilizar el glosario fue que "sacar un glosario en medio de una interacción sería poco profesional" (G1) y que "supondría una pérdida de tiempo y el cliente puede pensar que no estás suficientemente preparada para interpretar o que no vas a entender o explicar bien lo que él diga" (G5). Ante la futura interacción con un médico real, los alumnos eran más conscientes de la imagen que iban a proyectar y se preocupaban por que esta fuera lo más profesional posible.

\section{Aspectos pragmáticos}

La precisión terminológica es una de las mayores dificultades a las que se enfrenta el alumnado de ISP. Por esta razón se informaron sobre la posibilidad de llevar tabletas o gadget tecnológicos a la interacción, y en particular, sobre la posibilidad de entrar con ellos en la unidad de cuidados intensivos. “Encontramos que la utilización de este tipo de aparatos sí que está permitida desde 2010. Sin embargo, antes de esta fecha estaba prohibido por las perturbaciones que pudieran causar en algunos aparatos del hospital y en estimuladores cardíacos que llevan algunos pacientes. Hoy en día, las interferencias causadas por los aparatos tales como móviles o tablets ya no afectan a los dispositivos médicos si se encuentran a una distancia superior a un metro y medio, por lo que decidimos explotarlo como herramienta de trabajo" (G4). Este grupo no utilizó un glosario en papel, lo que facilitó la búsqueda de términos y agilizó el proceso de documentación.

Por otra parte, ningún grupo se documentó sobre aspectos culturales que pudieran afectar a la interacción, manifestando un desconocimiento evidente sobre el perfil de los pacientes que podrían encontrar en los contextos descritos. Tal y como recoge Sales (2005), "mediar no implica solo traducir las palabras, sino que va más allá, abarcando todos los aspectos de la comunicación no verbal lolor, gestos, movimientos del cuerpo, silencios, etc.), que culturalmente son claves" (p. 4). El mediadorintérprete debe tener siempre en mente elementos sobre la cultura y la sociedad de la que es originario el paciente: estructura familiar, religión, concepción de la salud o la enfermedad, entre otros. Por eso, necesita formarse continuamente, "documentándose de forma contrastada y especializada" (Sales, 2005, p. 5), algo que nuestros alumnos no hicieron y que tuvieron consecuencias en los role plays simulados, como veremos en el apartado siguiente.

\section{ANÁLISIS DE LA INTERACCIÓN Valoración de la preparación}

A pesar de que la mayoría de grupos consideró su preparación de las sesiones como buena (6 grupos) y uno de ellos como muy buena, lo cierto es que, luego de haber interactuado con el profesional, todos creyeron que podían haberla mejorado. La mayoría de eventuales mejoras que proponían se centraba en aspectos terminológicos o lingüísticos: haber incluido más datos en el glosario que habían 
elaborado (fotografías, explicaciones), más términos o también haberlos ordenado de forma distinta latendiendo a la especialidad, y dentro de cada una de ellas, a la temátical. No obstante, las mejoras también se centraban en aspectos socioculturales: haber indagado más en algunas particularidades culturales ${ }^{5}$ o en aspectos estratégicos. Respecto de este último, la consulta con un experto en la materia se presentó, a posteriori, como una fuente de información valiosa, pero no únicamente para cuestiones terminológicas, sino sobre todo para tener más conocimiento del contexto de trabajo: tipos de pacientes (edades, nacionalidades), patologías y consultas más frecuentes, situaciones en las que debían actuar o composición del equipo médico, entre otros.

Cuatro grupos confesaron que no les había servido de nada utilizar el glosario en la interacción: el nivel de estrés, la gesticulación excesiva de algunos alumnos y la falta de comprensión del término empleado por el médico, hicieron que tres de los cuatro grupos decidieran preguntar al profesional sanitario directamente y prescindir del glosario ${ }^{6}$.

Prefirieron pedir aclaraciones (en 25 ocasiones) y emplear paráfrasis a la hora de interpretar (en 8 ocasiones). En este sentido, la precisión terminológica no les pareció tan importante como la comprensión de los procesos (tratamientos, estado clínico etc.) una vez finalizada la sesión. No obstante, los tres grupos restantes manifestaron que la elaboración del glosario les había servido para familiarizarse con la terminología médica, y que lo consideraban una herramienta útil para la preparación de la sesión. También dos de los grupos consideraban el glosario como un "salvavidas" en el que apoyarse ( $G 1$ y G5), pues se sentían más seguros al saber que lo tenían con ellos. "No lo he utilizado finalmente, pero me daba más seguridad en mí misma saber que si me perdía o no entendía alguna palabra muy especializada, podía recurrir al glosario. Así, no me quedaría en blanco".

\section{Valoración de las cualidades interpretativas por parte del alumnado}

Una vez finalizada la sesión, el alumnado valoró las competencias que consideraban más importantes a la hora de realizar ISP en el ámbito médico-sanitario. En una escala Likert de 0 a 5, debían puntuar las cualidades que habían puesto en práctica durante la interacción con el profesional en función de su importancia en este contexto.

Como vemos en la Figura 1, todas las cualidades han superado la nota media de $3 / 5$, lo que se justifica porque la ISP en el ámbito sanitario es una de las especialidades consideradas más difíciles por los alumnos, eso sí, por debajo de la interpretación judicial (Sanz-Moreno, en prensa). Para todos los grupos, el secreto profesional y el conocimiento cultural fueron las cualidades más valoradas (4.42 de 5), seguidos por la imparcialidad (4.28 de 5). Esto se explica por los role plays que se representaron en clase y en los que el médico ordenaba al alumno informar a los familiares sobre el diagnóstico del paciente o los familiares-actores solicitaban esa información al intérprete, en ausencia del

\footnotetext{
5 Uno de los casos prácticos abordaba un posible trasplante de órganos de un paciente musulmán; otro versaba sobre una paciente senegalesa que se negaba a mirar a los ojos a la ginecóloga. En otro caso, el marido de una paciente, ambos de origen marroquí, exigía la presencia de personal médico femenino para un reconocimiento.

6 El visionado y análisis posteriores de la interacción con los médicos que se grabó en vídeo les ayudó a analizar su lenguaje corporal, lo que enriqueció sobremanera las autovaloraciones y el análisis crítico que realizaron de las sesiones.
} 


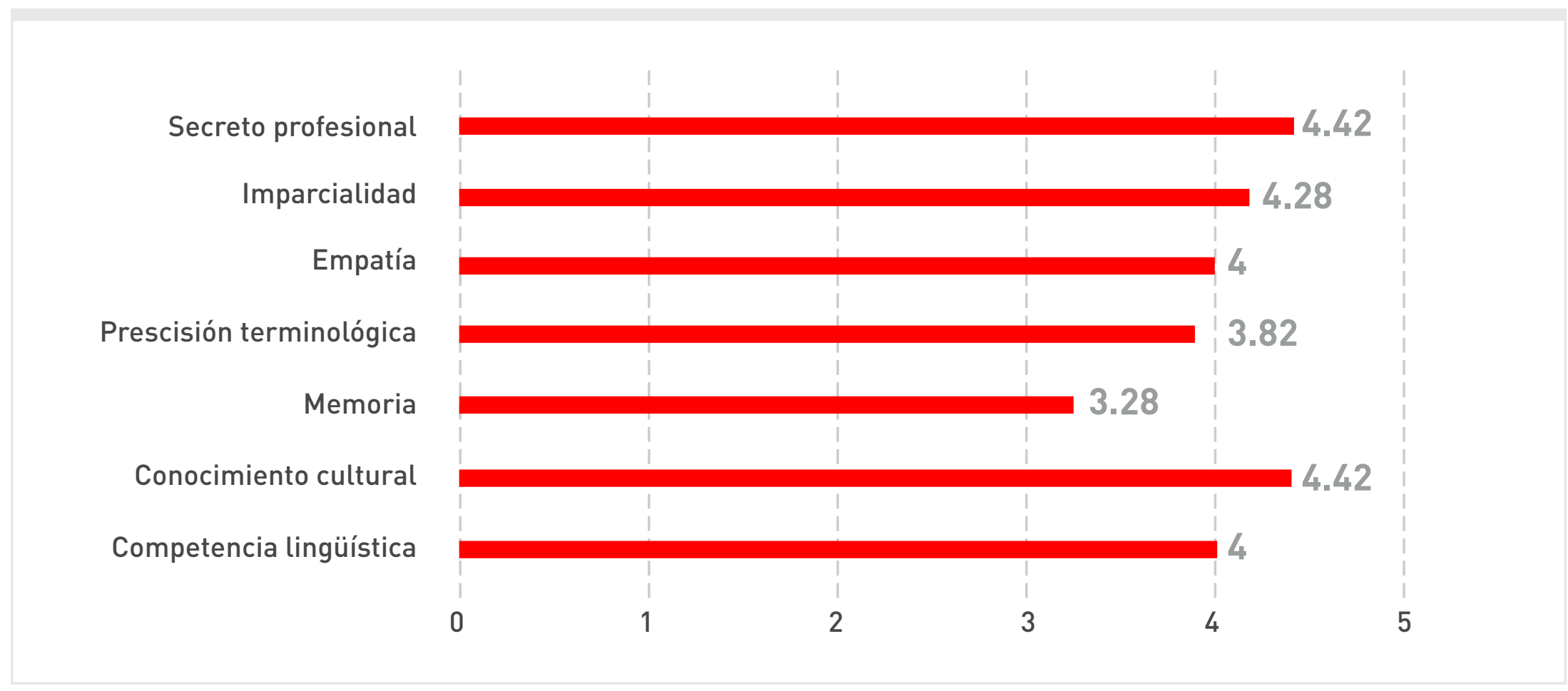

Figura 1. Evaluación de las cualidades del intérprete en ISP en el ámbito sanitario

facultativo. En ambos casos, el alumno hacía lo que se le pedía, sin reflexionar sobre las consecuencias de esa comunicación, y descartaba llevar la contraria al médico o negar la información al familiar. En el debate posterior, los alumnos manifestaron su confusión a la hora de abordar esta tarea, ya que desconocían la calidad y cantidad de información que podían/debían facilitar sin la presencia del médico. Otros pretendían tranquilizar a los familiares y dejar que el médico explicara el diagnóstico. El profesional médico explicó el significado y los límites del secreto profesional, basándose en su código deontológico, lo que nos permitió introducir la explicación teórica de la obligación del secreto profesional contemplada en los códigos deontológicos de los intérpretes (Asociación de Intérpretes de Conferencias de España [AICE], 2012; Asociación Española de Traductores, Correctores e Intérpretes [ASETRAD], s,f.; Asociación Profesional de Traductores e Intérpretes Judiciales y Jurados [APJIT], 2010). Del cuestionario posterior se desprende que los alumnos fueron conscientes del papel del mediador como figura central en la interacción, que debía adoptar decisiones y ser protagonista ly no resignarse a ser un actor secundario). "Me di cuenta de que no estamos solo para traducir palabras. Debemos asegurar que la comunicación se produzca, sea fluida y que el paciente esté lo mejor posible. Y para eso hay que hacer muchas más cosas" (G3).

También la imparcialidad fue abordada partiendo de dos situaciones: un caso en el que el familiar del paciente le pedía al intérprete que intercediera por él y convenciera al médico para que le dejara visitar a su familiar fuera del horario de visitas de la UCl; y el segundo, en el que la ginecóloga expresaba en voz alta su opinión sobre un embarazo no deseado de una inmigrante tunecina delante del intérprete y de la paciente. La alumna-intérprete del primer caso dudó entre una mayor intervención labogar por el familiar) o limitarse a transmitir el mensaje. Finalmente, hizo esto último. Su justificación era que entendía que la UCl dispone de unas normas para todos y que, por tanto, hacer una excepción podría entorpecer la dinámica de esta unidad. La alumna que actuaba de intérprete, en el segundo caso, tomó la iniciativa de explicarle a la médica cuál era su función y que no iba a entrar a juzgar a la paciente (añadiendo, además, que como profesional sanitario tampoco debería haberlo hecho). Parece que la 
combinación de la interacción con el médico y la sesión teórica dejaron huella en los alumnos que aplicaron sus conocimientos en este caso.

Únicamente dos de las cualidades que pedimos que valoraran no alcanzaron el 4/5: la memoria (3.28) y la precisión terminológica (3.82). Dado que los alumnos practicaron la interpretación de enlace, no consideraron la memoria como una destreza fundamental, ya que pactaron los turnos de palabra y la extensión de las intervenciones con carácter previo. Asimismo, el poder contar con el glosario y, sobre todo, preguntar al médico por el significado de algunos términos, hizo que los alumnos no se preocuparan en exceso por la precisión terminológica, y se centraran en que el paciente o el familiar entendieran los tratamientos o procesos. Además, ninguno de ellos tomó notas: primero, porque consideraron que las intervenciones eran cortas y no era necesario launque en 6 ocasiones tuvieron que pedirle al médico que repitiera la información), y en segundo lugar, porque no sabían cómo hacerlo. Esto último tiene su explicación porque en el role play en la UCI simulada, el médico les advirtió que las interacciones se realizaban, en el $80 \%$ de las ocasiones, de pie (algo en lo que no habían pensado). Esto dificultaba la toma de notas de los alumnos que, además, portaban sus glosarios.

En cuanto a las dificultades que tuvieron los alumnos (ver Figura 2), no ser imparcial es lo que más les preocupaba, ya que consideraron la imparcialidad como lo más difícil de conseguir (4.42 de 5), aunque también tenían cierto miedo a no comprender al profesional (4.14 de 5). Estos resultados pueden explicarse por los casos prácticos a los que se enfrentaron los alumnos que presentaban dilemas éticos luna paciente con un embarazo no deseado, otra con una enfermedad de transmisión sexual, un paciente en la $\mathrm{UCl}$ en coma del que se informa a los familiares sobre su idoneidad para trasplantar sus órganos, etc.). Esto explica igualmente la preocupación que la afectación psicológica podía provocar en el intérprete en formación, que también alcanzó una puntuación elevada, un 3/5, y que antes de la sesión ni siquiera se habían planteado.

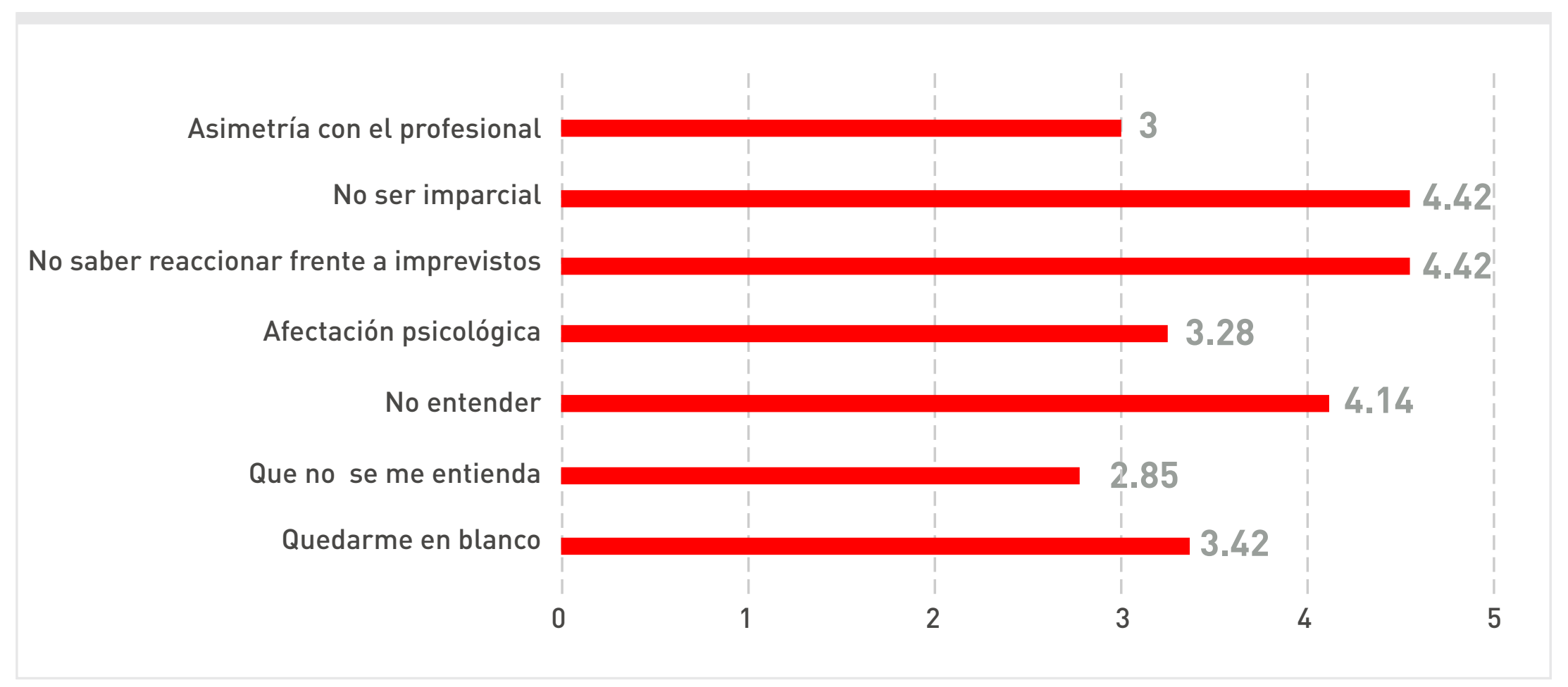

Figura 2. Evaluación de las dificultades del intérprete en ISP en el ámbito sanitario 


\section{CONCLUSIONES}

Contar con médicos en el aula de ISP es un privilegio que, estimamos, debemos seguir explorando. En primer lugar, porque interactuar con personas que desempeñan su labor en contextos reales favorece el conocimiento de primera mano por parte del estudiantado de situaciones a las que, en numerosos casos, es ajeno, puesto que la mayoría de los alumnos manifestaron un cambio de perspectiva después de la interacción con el profesional. Además, la documentación previa a la interacción se les presenta como algo indispensable, pero con un fin de preparación más que de utilización efectiva de los glosarios en la propia interacción con el paciente. Documentarse sobre aspectos más que lingüísticos, como las diferencias culturales, el perfil de paciente o de los familiares con los que deben trabajar, el emplazamiento físico del intérprete y la conformación del equipo médico son algunas cuestiones sobre las que los alumnos ni siquiera se habían parado a pensar antes de realizar los ejercicios de simulación con un médico. Además, acabaron otorgando más importancia a factores como la imparcialidad o la confidencialidad, y durante la interacción, desarrollaron estrategias de colaboración y trabajo en equipo con el profesional. Las encuestas que se realizaron al finalizar cada sesión demuestran que la socialización del alumno con el experto en la materia (Monzó, 2003) derivó en la adquisición de destrezas interpretativas y temáticas.

Una de las conclusiones más interesantes a las que llegaron los alumnos fue que, a pesar de que ambas sesiones se realizaron en el marco de la interpretación en el ámbito médico sanitario, había diferencias notables entre interpretar en una consulta ginecológica o en una UCl, no solo de funcionamiento del servicio, sino del perfil del paciente y del estado psicológico del mismo. Además, el role play favoreció interesantes debates sobre los límites de la implicación del intérprete en la interacción con el usuario, permitiendo la introducción de conceptos teóricos de la materia y facilitando la retroalimentación constante y necesaria entre teoría y práctica de la interpretación. Por otra parte, el hecho de que los profesionales médicos accedieran a participar en las sesiones de forma altruista hizo que los alumnos apreciaran su dedicación, su esfuerzo y su experiencia; pero esta admiración y respeto hacia el trabajo del profesional no conllevó una percepción consciente de la asimetría que tradicionalmente se puede observar en el intercambio comunicativo entre intérprete/paciente, sino que el alumno pasó a considerarlo como un compañero de trabajo con el que se podía cooperar. Su disposición a explicar y a contar con los alumnos en un plano colaborativo enriqueció los intercambios y contribuyó a que ambas sesiones fueran un rotundo éxito. 


\section{REFERENCIAS}

Alonso, I. \& Baigorri, J. (2008). Enseñar la interpretación en los servicios públicos: una experiencia docente. Redit, 1, 1-25. Recuperado de https:// goo.gl/KBcZfw

Angelelli C. (2008). The role of the interpreter in healthcare setting. A plea for a dialogue between research and practice. En C. Valero-Garcés, \& A. Martin (Eds.), Crossing Borders in Community Interpreting: Definitions and dilemas (pp. 147164). Amsterdam, Philadelphia: Benjamins.

Asociación de Intérpretes de Conferencias de España (AICE, 2012). Código deontológico. Recuperado de http://www.aice-interpretes.com/pdf/ codigo-deontologico.pdf

Asociación Española de Traductores, Correctores e Intérpretes (ASETRAD, s,f.). Código deontológico. Recuperado de https://asetrad.org/laasociacion/codigo-deontologico

Asociación Profesional de Traductores e Intérpretes Judiciales y Jurados (APJIT, 2010). Código deontológico para intérpretes y traductores judiciales y jurados. Recuperado de http://www.aptij.es/img/doc/CD\%20APTIJ.pdf

Baigorri, J. (dir), Alonso, I., Camas R., Martín, O., Otero, C., Palacio, E. \& Russo, M. (2006). Materiales didácticos para la enseñanza de la interpretación en el ámbito social (Alemán-francés-inglés- Italiano-español). Salamanca: Universidad de Salamanca.

Cambridge, J. (2002). Interlocutor roles and the pressures on interpreters. En C. Valero-Garcés, \& G. Mancho Barés (Eds.). Traducción e Interpretación en los Servicios Públicos: Nuevas Necesidades para Nuevas Realidades. Community interpreting and translating new needs for new realities (pp. 121-126). Alcalá de Henares: Universidad de Alcalá.

Grupo CRITT (2014). La práctica de la mediación interlingüística e intercultural en el ámbito sanitario. Granada: Comares.

Del Pozo, I. (2009). Una propuesta formativa en interpretación en los servicios públicos en el marco del EEES. Redit, 3, 31-51. Recuperado de https://goo.gl/DW6Zh1

Fernández Pérez, M. (2011). Variedades de exercicios de roleplay como ferramenta didáctica na formación de intérpretes para os servizos públicos. En S. Cruces Colado, M. del Pozo Triviño, A. Luna Alonso \& A. Álvarez Lugrís (Eds.), Traducir en la frontera (pp. 161-169). Granada: Atrio.

Fernández Pérez, M. (2015). Designing role-play Models for Telephone Interpreting Training. MonTI. Monografías de Traducción e Interpretación, núm. esp. 2, 259-279. doi: http://dx.doi.org/10.6035/MonTI.2015.ne2.10

Gallego, D. \& Tolosa M. (2012) Terminología bilingüe y documentación ad hoc para intérpretes de conferencias. Una aproximación metodológica basada en corpus. Estudios de Traducción, 2, 33-46. doi: http://dx.doi.org/10.5209/rev_ESTR.2012.v2.38976

García-Beyaert, S. (2013). Código deontológico. Principios éticos y pautas de buenas prácticas para la interpretación en los servicios públicos y el ámbito comunitario. Recuperado de http://www.l-in-k.org/Codigo_deontologico_ISPC.pdf

Gonzalo García, C., Fraile, E. \& Pérez, E. (2005). Selección y evaluación de recursos informativos en Internet para el traductor literario. En C. Gonzalo García \& V. García Yebra, (Eds.), Manual de documentación para la traducción literaria, (pp. 201-243). Madrid: Arco Libros.

Jiménez Ivars, A. (2012). Primeros pasos hacia la interpretación Inglés-Español (Guía didáctica). Castellón: Universitat Jaume I.

Jiménez Ivars, A. \& Hurtado Albir A. (2003). Variedades de traducción a la vista. Definición y clasificación. TRANS, 7, 47-57. Recuperado de https:// goo.gl/zW3ujt

Lázaro, R. (abril 2007). Dilemas éticos del traductor/intérprete en los servicios. Ponencia presentada en el 1st International Forum on Translation/ Interpreting and Social Activism, Granada, España. Recuperado de http://www.translationactivism.com/articles/Raquel_Lazaro.pdf

Lázaro, R. \& Pena, C. (2010). Training interpreters in the healthcare area: guidelines for the paediatrics consultation. En R. Caballero Rodríguez

\& M. J. Pinar Sanz (Eds.) Ways and modes of human communication. Cuenca: Ediciones de la Universidad de Castilla-La Mancha.

Martin, A. (2014). Interpretación social: rasgos, dilemas y profesionalización. La linterna del Traductor, 9. Recuperado de https://goo.gl/B7yM9D

Medlnt (2008). Medlnt: Developing a curriculum for medical interpreters. Recuperado de https://goo.gl/QfrQdR

Montalt, V. (2005). El género como espacio de socialización del estudiante de traducción científico-técnica. En I. García Izquierdo (Ed.), El género textual y la traducción. Reflexiones teóricas y aplicaciones pedagógicas (pp. 19-36). Berna: Peter Lang.

Monzó, E. (2003). Las socializaciones del traductor especializado: El papel de los géneros. Revista de la Facultad de Lenguas Modernas, 6, 15-29.

Niska, H. (2002). Community interpreter training. Past, present, future. En G. Garzone \& M. Viezzi. (Eds.). Interpreting in the 21st century (pp. 133158). Amsterdam/Philadelphia: John Benjamins.

Ozolins, U. (2013). Role playing "Pumpkin”. En C. Schäffner, K. Kredens \& Y. Fowler (Eds.). Interpreting in a changing landscape (pp. 31-43). Amsterdam: John Benjamins.

Ruiz Rosendo, L. (2007). Propuesta de formación en interpretación en el ámbito de la medicina. Panacela, 9(26), 173-182. Recuperado de https:// goo.gl/8MjXxs 
Sales, D. (2005). Panorama de la mediación intercultural y la traducción/interpretación en los servicios públicos en España. Translation Journal, 9(1). Recuperado de https://goo.gl/FW08Xk

Sanz-Moreno, R. (en prensa). El profesional del Derecho en la formación del intérprete. Una experiencia didáctica. Revista d'Innovació Docent Universitària, 10

Valero-Garcés, C. (2005). Terminology and Ad hoc Interpreters in Public Services. An Empirical Study, JoSTrans, 3, 75-96. Recuperado de https:// goo.gl/eujj1T

Valero-Garcés, C. (2006). Formas de mediación intercultural. Traducción e Interpretación en los servicios públicos. Conceptos, datos, situaciones y práctica. Granada: Comares.

Vitalaru, B. \& Lázaro R. (2011). FITISPos-UAH: Formación y práctica en Traducción e Interpretación sanitaria. En R. Lázaro (Ed.) 2011. Traducción e Interpretación en los Servicios Públicos en el siglo XXI. Avanzando hacia la unidad en medio de la globalización (pp.84-94). Alcalá de Henares: Universidad de Alcalá de Henares.

Wadensjö, C. (1995). Dialogue Interpreting and the Distribution of Responsibility. Hermes Journal of Linguistics, 14, 11-129. Recuperado de https://goo.gl/qN7ZyB

(c) Los autores. Este artículo es publicado por la Revista Digital de Investigación en Docencia Universitaria del Área de Institutional Research and Effectiveness de la Dirección de Aseguramiento de la Calidad, Universidad Peruana de Ciencias Aplicadas. Este es un artículo de acceso abierto, distribuido bajo los términos de la LicenciaCreativeCommons Atribución-Compartirlgual 4.0 Internacional.I http://creativecommons.org/licenses/bysa/4.0/I, que permite el uso no comercial, distribución y reproducción en cualquier medio, siempre que la obra original sea debidamente citada. 


\section{APÉNDICE A}

Modelo de plantilla de evaluación de recursos electrónicos (adaptado de Gonzalo García, Fraile \& Pérez, 2005)

- Título

- URL

- Tipo de fuente o recurso

- Lengua

- Responsabilidad principal

- Lugar de procedencia

- Contacto/webmaster

- Descripción del contenido y utilidad de la información

- Diseño y presentación de la información

- Estrategias de navegación

- Presencia y calidad de enlaces externos

- Áreas de aplicación (finalidad del sitio web)

- Fecha de creación del sitio web

- Fecha de la última actualización del sitio web

- Puntuación (del 1 al 5)

\section{APÉNDICE B}

\section{CUESTIONARIO}

1. Valora las destrezas y habilidades que consideras más importantes a la hora de abordar este tipo de interpretación.

\begin{tabular}{|l|l|l|l|l|l|}
\hline Quedarme en blanco & 1 & 2 & 3 & 4 & 5 \\
\hline Competencia lingüística & & & \\
\hline Competencia lingüística & & \\
\hline Conocimiento cultural & & \\
\hline Curiosidad & & \\
\hline Memoria & & \\
\hline Precisión terminológica & & \\
\hline Empatía & & \\
\hline Imparcialidad & & \\
\hline
\end{tabular}


2. Valora lo que consideras que te preocupa más a la hora de abordar esta interpretación

\begin{tabular}{|l|l|l|l|l|l|}
\hline Quedarme en blanco & 1 & 2 & 3 & 4 & 5 \\
\hline Hacer el ridículo & & & \\
\hline Que no se me entienda & & \\
\hline No entender al médico o al paciente & & \\
\hline Que me afecte psicológicamente & & \\
\hline No encontrar la palabra adecuada & & \\
\hline No saber cómo reaccionar frente a imprevistos & & \\
\hline No ser imparcial & & \\
\hline La asimetría respecto del profesional & & \\
\hline
\end{tabular}

DOCUMENTACIÓN. Preparación previa

3. ¿Conocías la plantilla de evaluación de recursos electrónicos de Gonzalo García (2015)? Sí

No

NS/NC

4. ¿La has encontrado útil?

Sí

No

NS/NC

5. ¿Por qué?

6. ¿Cómo valoras tu preparación de la sesión?

Mala

Regular

Buena

Muy buena

NS/NC

7. ¿En qué crees que podrías haber mejorado tu preparación?

8. ¿Has utilizado (o hubieras utilizado) el glosario?

Sí

No

$\mathrm{NS} / \mathrm{NC}$ 
9. ¿Te ha servido (o hubiera servido) el glosario?

Sí

No

NS/NC

10. ¿Cómo?

\begin{tabular}{|l|l|l|l|l|l|l|}
\hline Me ha dado más seguridad llevarlo & 1 & 2 & 3 & 4 \\
\hline Al prepararlo, me he familiarizado & & \\
\hline con la terminología & & \\
\hline Me ha servido para afianzar mis respuestas & \\
\hline Me ha permitido ser más preciso & \\
\hline Me he sentido más profesional \\
\hline Me ha permitido ganar tiempo mientras buscaba \\
\hline un término y tranquilizarme
\end{tabular}

11. ¿Cuáles han sido las ventajas de trabajar en grupo?

\section{2. ¿Y los inconvenientes?}

Traducción a la vista

\section{Valora tu TAV}

Mala

Regular

Buena

Muy buena

NS/NC

\section{4. ¿Por qué?}

15. ¿Qué tipo de errores crees que has cometido? (puedes marcar más de una respuesta) Omisión

Adición

Reformulación (Ej: falsos comienzos)

Sinsentidos 
Contrasentidos

Incorrecciones gramaticales (Ej: Las amigos)

Incorrecciones terminológicas

16. ¿Qué tipo de error crees que has evitado con la preparación de la sesión?

17. Valora la sesión de interpretación en el ámbito sanitario

18. Valora al ponente de la sesión (Dr. Rafa Faus/Dra. Esther Ortiz)

19. ¿Ha cambiado en algo tu opinión sobre este tipo de interpretación? ¿Te atreverías ahora a hacer una?

Expresa tu opinión sobre la utilidad de estas clases y, en particular, de esta sesión (con preparación previa). 